\title{
Pocket-Sized Transthoracic Echocardiography Device for the Measurement of Cardiac Chamber Size and Function
}

\author{
Shota Fukuda, MD; Kenei Shimada, MD; Toshihiro Kawasaki, RDCS; Hiromi Fujimoto, RDCS; \\ Kumiko Maeda, RDCS; Hitoshi Inanami, MD; Ken Yoshida, MD; Satoshi Jissho, MD; \\ Haruyuki Taguchi, MD; Minoru Yoshiyama, MD*; Junichi Yoshikawa, MD
}

\begin{abstract}
Background: A pocket-sized portable transthoracic echocardiographic (pTTE) imaging device is commercially available, but its feasibility and accuracy in the assessment of cardiac chamber size and function has not been fully compared with the results of standard TTE (sTTE) examination.

Methods and Results: The target population comprised 125 unselected patients who underwent sTTE and pTTE examinations. The left ventricular (LV) diastolic and systolic dimensions, fractional shortening (FS), the thickness of the interventricular septum (IVS) and of the LV posterior wall (PWT), left atrial (LA) dimension, and ascending aorta diameter were measured. Echocardiographic measurements were completed for both pTTE and sTTE in all patients (feasibility 100\%). LV dimensions, FS, IVS, PWT, LA dimension, and aorta diameter obtained by pTTE showed excellent correlation and agreement with sTTE $(r=0.87-0.98$, all $\mathrm{P}<0.001)$. Observer variabilities for these measurements were similar between pTTE and sTTE.

Conclusions: In the present study, pTTE with the Acuson P10 was feasible and accurate for assessing cardiac chamber size and function. (Circ J 2009; 73: 1092-1096)
\end{abstract}

Key Words: Echocardiography; Imaging; Ventricular function

$\mathbf{T}$ echnological advances in electronic miniaturization have resulted in the creation of small, portable transthoracic echocardiographic (pTTE) devices. The major advantage of a portable device is being able to provide immediate information both within and, for the first time, outside the hospital setting. Previous studies have reported that pTTE is superior to physical examination, and comparable with complete standard TTE (sTTE) as a diagnostic tool in clinical practice!-3 Physical examination is recognized as an initial screening method, but the skill set required to perform physical examinations has unfortunately deteriorated over the past 2 decades as medical technology has advanced-6 Therefore, visualizing the heart as well as being able to calculate limited quantitative measurements of the cardiac chambers and their function, may result in improved patient care and/or understanding.

Compared with a stethoscope, previous portable devices were too large and heavy $(\approx 2.5 \mathrm{~kg})$ for physicians to carry while making rounds. A pocket-sized imaging device has better portability and agility, enhancing the use of pTTE? We investigated the feasibility and accuracy of pTTE for assessing cardiac chamber size and function.

(Received November 18, 2008; revised manuscript received January 27, 2009; accepted January 28, 2009; released online April 16, 2009) Department of Medicine, Cardiovascular Division, Osaka Ekisaikai Hospital, *Department of Internal Medicine and Cardiology, Osaka City University School of Medicine, Osaka, Japan

Mailing address: Shota Fukuda, MD, Department of Medicine, Cardiovascular Division, Osaka Ekisaikai Hospital, 2-1-10 Honden, Nishiku, Osaka 550-0022, Japan. E-mail: h-syouta@mve.biglobe.ne.jp All rights are reserved to the Japanese Circulation Society. For permissions, please e-mail: cj@j-circ.or.jp

\section{Methods}

\section{Study Population}

The study group consisted of 125 unselected patients (70 males, mean age $70 \pm 13$ years) who were scheduled for sTTE to evaluate left ventricular (LV) function: 65 patients with coronary artery disease ( 24 with a history of myo-
\end{abstract}

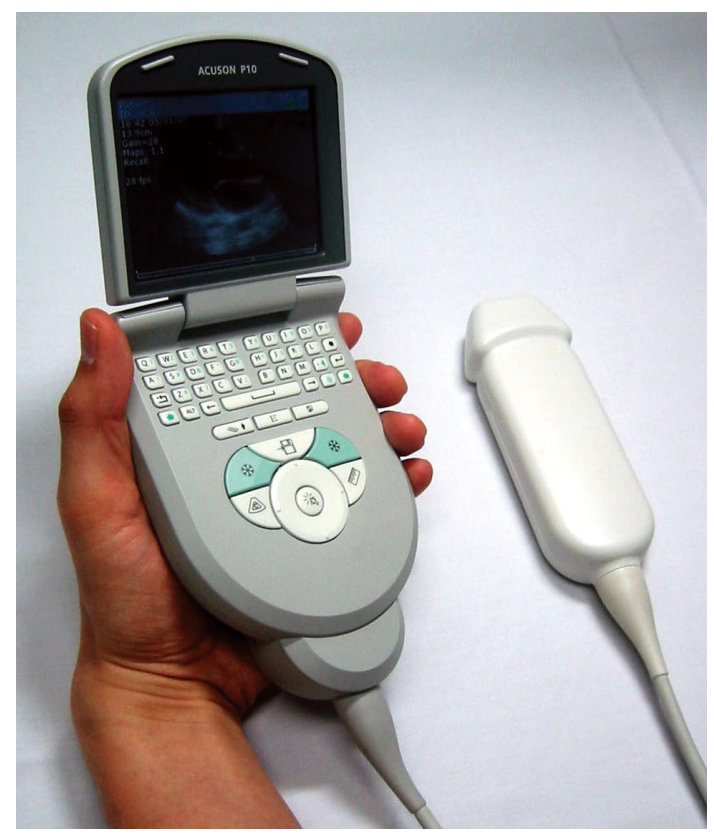

Figure 1. The pocket-sized transthoracic echocardiography device, Acuson P10, showing the body of the system (Left) and the transducer (Right). 


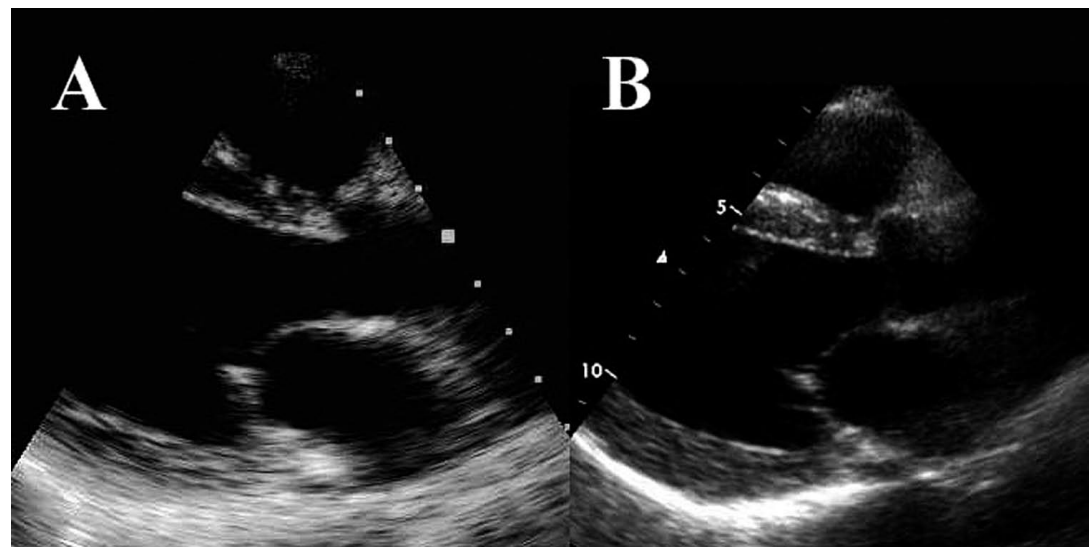

Figure 2. Parasternal long-axis view: (A) portable transthoracic echocardiography and (B) standard transthoracic echocardiography.

cardial infarction), 21 patients with hypertension, 11 with valvular heart disease, 8 with dilated or hypertrophic cardiomyopathy, 10 with pulmonary hypertension, and 10 with arrhythmia. Initially, 90 patients had pTTE immediately after sTTE. Both echocardiographic examinations were done in the echocardiographic laboratory by expert sonographers. In the later 35 patients, pTTE was performed by the physician at the bedside and those patients had their sTTE examination in the echocardiographic laboratory $3 \pm 4$ days later. Stable hemodynamic conditions were confirmed during this period.

Commercially available echocardiographic systems were used for the sTTE examination, including Sequoia 512 (Siemens Medical Solutions, Mountainview, CA, USA), Aplio SSA-770 (Toshiba Medical Systems, Tokyo, Japan), and Vivid 7 (GE Medical Systems, Milwaukee, WI, USA). This study was approved by the Ethics Committee of the Osaka Ekisaikai Hospital.

\section{pTTE}

The pTTE was performed with the Acuson P10 (Siemens Medical Solutions) (Figure 1), which has a 64-element phased array transducer with a frequency range of $2-4 \mathrm{MHz}$. The device has tissue harmonic imaging, as well as automatic time gain compensation and digital storage capabilities. The total weight of the system, including the transducer, 3.7 inch display and control panel, is $0.725 \mathrm{~kg}$. The size of the unit and transducer are $56 \mathrm{~mm}$ and $33 \mathrm{~mm}$ in height, $97 \mathrm{~mm}$ and $48 \mathrm{~mm}$ in width, and $145 \mathrm{~mm}$ and $136 \mathrm{~mm}$ in length, respectively. The rechargeable batteries are fully charged in $2.5 \mathrm{~h}$ ( $75 \%$ charge in $1 \mathrm{~h}$ ), and the set-up time for the image acquisition is less than $10 \mathrm{~s}$. The device has measurement packages that include linear measurement caliper capabilities.

The parasternal long-axis view was obtained with pTTE and sTTE (Figure 2). The following echocardiographic parameters were measured according to the recommendation of the American Society of Echocardiography: 8 LV enddiastolic and end-systolic dimensions (LVDd, LVDs), the thickness of the basal interventricular septum (IVS) and posterior wall (PWT) at end-diastole, left atrial dimension (LAd), and ascending aorta diameter. LV fractional shortening (FS) was then calculated as (diastolic dimensionsystolic dimension)/diastolic dimension $\times 100$.

In addition, the presence or absence of $\mathrm{LV}$ regional wall motion abnormalities (RWMA) and pericardial effusion were assessed by pTTE and sTTE, respectively. RWMA was defined as a segment with hypokinesis, akinesis or dyskinesis. The pericardial effusion was diagnosed as an echo-free space within the pericardial layers.

\section{Statistical Analysis}

Continuous values are expressed as mean \pm standard deviation (SD). Comparisons between 2 groups for the parametric data were made using the t-test. Correlations between the sTTE and pTTE results were obtained using least squares fit linear regression analysis. Differences were considered significant at $\mathrm{P}<0.05$. Differences between sTTE and pTTE results were also compared with the mean values obtained by sTTE and pTTE using the Bland-Altman method, with the limits of agreement defined as $\pm 2 \mathrm{SD}$ of the difference between the 2 methods.

Inter-observer variability for the LV dimensions, FS, IVS, PWT, LAd, and aorta diameter was obtained by analysis of 30 random patients by 2 independent blinded observers. Intra-observer variability was performed by analysis of the other 30 patients by the same observer at 2 different time points. The results were analyzed by the Bland-Altman method.

\section{Results}

Echocardiographic measurements were completed for pTTE and sTTE in all patients (feasibility 100\%). The range of echocardiographic parameters by sTTE was $31-67 \mathrm{~mm}$ for LVDd, 19-60 mm for LVDs, 10-51\% for FS, 7-15 mm for IVS, 7-15 mm for PWT, 20-59 mm for LAd, and 20$38 \mathrm{~mm}$ for aorta diameter. In the 90 patients who had pTTE and STTE performed in the echocardiographic laboratory, correlations between the measurements by pTTE and sTTE were excellent (Figure 3) and there were no significant differences between them for any echocardiographic parameter. Bland-Altman plots demonstrated small systematic differences, or mean bias, between the pTTE and sTTE measurements, with close limits of agreement (Figure 4).

In the 35 patients who had pTTE performed at the bedside by the physician, there were excellent correlations between the measurements by pTTE and sTTE for LVDd $(r=0.97)$ and LVDs $(\mathrm{r}=0.98)$, FS $(\mathrm{r}=0.93)$, IVS $(\mathrm{r}=0.90)$, PWT $(\mathrm{r}=$ $0.88)$, LAd $(\mathrm{r}=0.93)$, and aorta diameter $(\mathrm{r}=0.85)$ (all $\mathrm{P}<0.001$ ). Bland-Altman analysis showed small systematic differences and limits of agreement between pTTE and STTE for LVDd $(-0.3 \mathrm{~mm}, 1.1 \mathrm{~mm})$ and LVDs $(0.1 \mathrm{~mm}$, $1.0 \mathrm{~mm})$, FS $(-0.7 \%, 2.7 \%)$, IVS $(0.1 \mathrm{~mm}, 0.6 \mathrm{~mm})$, PWT $(0.2 \mathrm{~mm}, 0.6 \mathrm{~mm}), \mathrm{LAd}(0.0 \mathrm{~mm}, 1.4 \mathrm{~mm})$, and aorta diameter $(0.1 \mathrm{~mm}, 1.1 \mathrm{~mm})$. These values were comparable with the results for sTTE and pTTE obtained in the echocardio- 


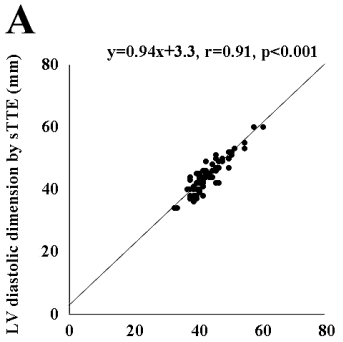

LV diastolic dimension by pTTE $(\mathrm{mm})$

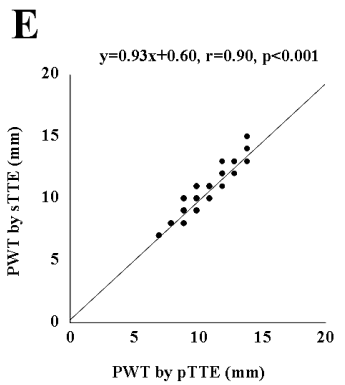

B

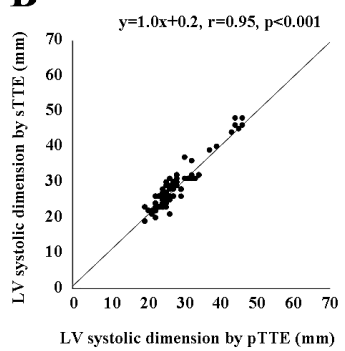

$\mathbf{F}$

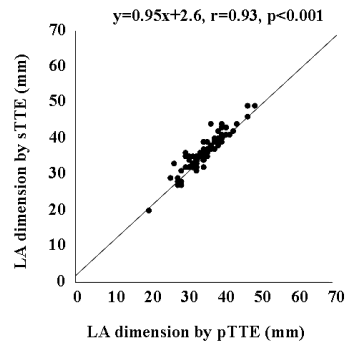

C

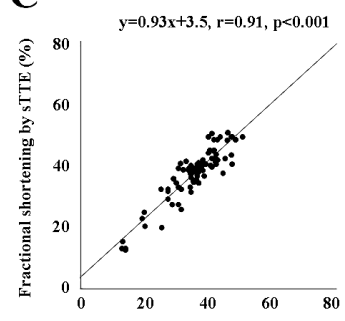

Fractional shortening by pTTE (\%)

G

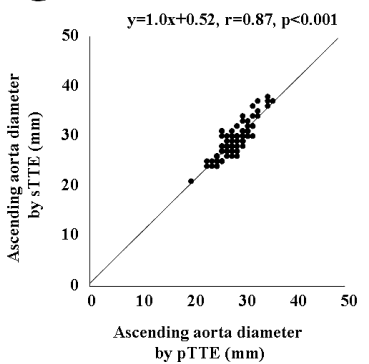

D

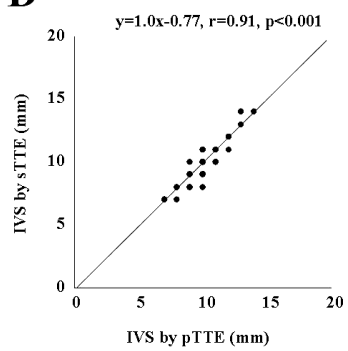

Figure 3. Regression plots showing the correlation between portable transthoracic echocardiography (pTTE) and standard transthoracic echocardiography (sTTE) measurements for left ventricular (LV) diastolic (A) and systolic (B) dimensions, fractional shortening (C), interventricular septum (IVS, D), posterior wall thickness (PWT, E), left atrial (LA) dimension $(\mathbf{F})$, and aorta diameter $(\mathbf{G})$. The solid line represents the regression lines between the pTTE and sTTE measurements.

A

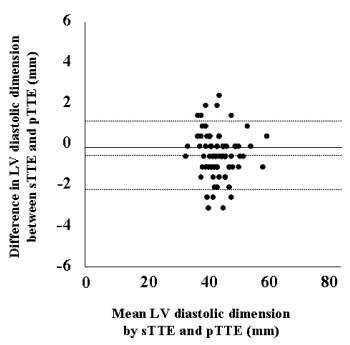

$\mathbf{E}$

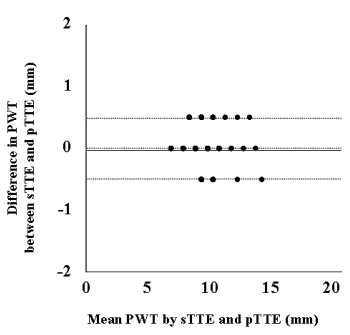

B

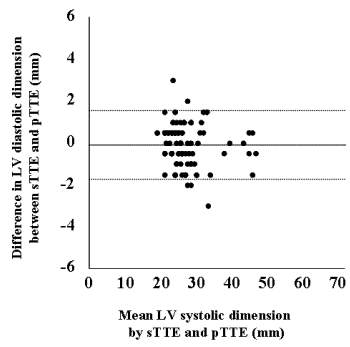

F

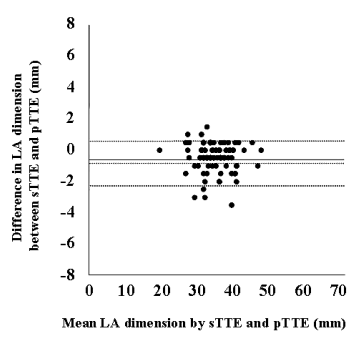

C

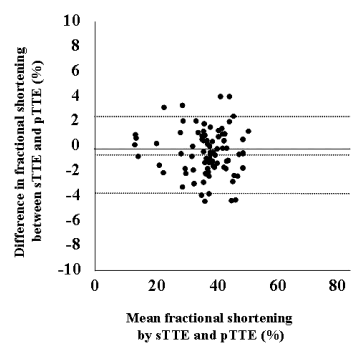

G

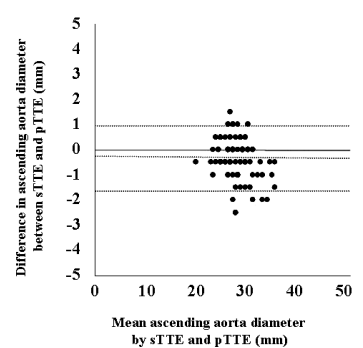

D

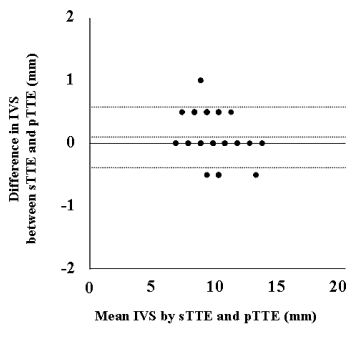

Figure 4. Scatterplot of the differences in the left ventricular (LV) diastolic (A) and systolic (B) dimensions, fractional shortening (C), interventricular septum (IVS, D), posterior wall thickness (PWT, E), left atrial (LA) dimension (F), and aorta diameter $(\mathbf{G})$ between portable transthoracic echocardiography and standard transthoracic echocardiography, showing the mean difference and the limits of agreement (solid lines). pTEE, portable transthoracic echocardiography; sTEE, standard transthoracic echocardiography.

graphic laboratory (Figures 3,4).

In the total patients included in this analysis, sTTE showed RWMA in $33(26 \%)$. There was concordance between sTTE and pTTE for the presence or absence of RWMA in $116(93 \%)$ of 125 patients. The sensitivity and specificity of pTTE for detecting RWMA was $88 \%$ and $95 \%$, respectively. Pericardial effusion was detected by sTTE and pTTE in $5(4 \%)$ of 125 patients.

Observer variabilities were similar between the pTTE and sTTE measurements for the LV dimensions, FS, IVS, PWT, LAd, and aorta diameter (Table). 
Table. Intra- and Interobserver Variabilities in Echocardiographic Measurements

\begin{tabular}{llllllll}
\hline & LVDd & LVDs & FS & IVS & PWT & LAd $\begin{array}{c}\text { Ascending } \\
\text { aorta diameter }\end{array}$ \\
\hline $\begin{array}{l}\text { Intra-observer variability } \\
\quad \text { sTTE }\end{array}$ & $0.7 \mathrm{~mm}$ & $0.8 \mathrm{~mm}$ & $1.4 \%$ & $0.2 \mathrm{~mm}$ & $0.2 \mathrm{~mm}$ & $0.7 \mathrm{~mm}$ & $0.8 \mathrm{~mm}$ \\
$\quad \begin{array}{l}\text { pTTE } \\
\text { Inter-observer variability }\end{array}$ & $0.8 \mathrm{~mm}$ & $1.1 \mathrm{~mm}$ & $2.0 \%$ & $0.3 \mathrm{~mm}$ & $0.4 \mathrm{~mm}$ & $1.1 \mathrm{~mm}$ & $1.2 \mathrm{~mm}$ \\
$\quad$ sTTE & $0.8 \mathrm{~mm}$ & $1.0 \mathrm{~mm}$ & $2.6 \%$ & $0.3 \mathrm{~mm}$ & $0.3 \mathrm{~mm}$ & $0.9 \mathrm{~mm}$ & $1.1 \mathrm{~mm}$ \\
$\quad$ pTTE & $1.2 \mathrm{~mm}$ & $1.1 \mathrm{~mm}$ & $2.6 \%$ & $0.4 \mathrm{~mm}$ & $0.4 \mathrm{~mm}$ & $1.0 \mathrm{~mm}$ & $1.8 \mathrm{~mm}$ \\
\hline
\end{tabular}

LVDd, left ventricular diastolic dimension; LVDs, left ventricular systolic dimension; FS, fractional shortening; IVS, interventricular septum; PWT, posterior wall thickness; LAd, left atrial dimension; sTTE, standard transthoracic echocardiography; pTTE, portable transthoracic echocardiography.

\section{Discussion}

This study demonstrated that the recently introduced pocket-sized echocardiography device can quantify cardiac chamber size and function in the clinical setting.

TTE is the standard imaging modality for assessing cardiovascular anatomy, function and physiology in clinical practice ${ }^{10-13}$ An expensive high-end instrument is used in the echocardiographic laboratory, operated by a skilled sonographer and supervised by experienced physicians. Echocardiographic examination is, therefore, performed several days after the initial physician-patient encounter. However, the indication for TTE depends on the findings of a physical examination, together with patient interview. Limitations in physical examination skills required to diagnose cardiovascular abnormalites have been documented, resulting in misleading diagnostic evaluations, therapeutic plans or patient prognoses ${ }^{4-6}$ Furthermore, skills in physical examination have declined over the past 2 decades.

Recent advances in electronics have enabled the creation of small, portable imaging devices. Bruce et $\mathrm{al}^{1}$ and Vourvouri et $\mathrm{al}^{2}$ reported excellent correlations between pTTE and sTTE in the measurement of aortic diameter and LV mass. Spencer et al demonstrated that physical examination failed to detect $59 \%$ of all cardiovascular conditions and $43 \%$ of major findings, which was reduced to $21 \%$ by pTTE? Previous cost-effectiveness analyses suggest that when pTTE is used as the initial screening of patients with suspected cardiac disease, there is approximately $20 \%$ reduction in sTTE indication! ${ }^{14,15}$ Another potential application of pTTE is a limited and focused echocardiographic examination. In the follow-up of many cardiac abnormalities, a limited imaging protocol (ie, few image acqusitions in a short time) is adequate to answer referral questions in clinical practice!6,17 Limited quantitative measurements, such as cardiac chamber size and function as assessed in the present study, are suitable and sufficient for a limited and focused echocardiographic examination!8

The Acuson P10 has recently become commercially available, potentially furthering the construction of a personal imager? We showed excellent correlations between pTTE and STTE for the measurements of cardiac chamber size and function in 125 unselected patients, even with the small monitor size and limited image setting. Furthermore, pTTE detected RWMA and pericardial effusion, which are common reasons in clinical practice for referring the patient to sTTE. Therefore, pTTE with the Acuson P10 can be used for initial screening to indentify or exclude cardiac abnormalites, or in the follow-up of cardiac diseases. The Acuson P10 is able to be used anywhere as part of the clinical examination. Physicians can carry a pTTE device while making rounds, just like a stethoscope. From the practical point of view, pTTE with Acuson P10 may be easier to use than the conventional portable devices available during hospitalization of inpatients, as well as outside the hospital setting, including the management of home-care patients. Consequently, there may be widespread use of pTTE as a diagnostic tool supplementing the physical examination, which would strengthen the accuracy of diagnosing various cardiac diseases in clinical practice.

\section{Study Limitations}

First, the image quality of the Acuson P10 did not equal that of high-end instruments and the device does not have color Doppler capability. Therefore, pTTE should not replace sTTE and physical examinations 19,20

Second, the datasets were acquired and analyzed by expert sonographers or physician with $>2$ years of experience in echocardiography with $>800$ examinations. In the present study 90 patients had pTTE performed in the echocardiographic laboratory by a sonographer, and 35 patients had it performed at the bedside by a physician. Similar correlations between the pTTE and sTTE measurements were obtained for different protocols, suggesting that accurate measurement with pTTE relies on experience in echocardiography rather than where it is performed. In fact, skills in acquiring and interpreting the pTTE images are recommended, even though the device is small in size and easy to use ? $^{21}$

Third, unselected patients were enrolled because this study was initially focused on investigating the ability of pTTE to measure cardiac chamber size and function in the clinical setting. We included 33 patients with RWMA, and the ability of pTTE to detect RWMA was demonstrated. However, it remains unclear whether pTTE has the ability to assess other reasons for patient referral for sTTE, because of the relatively small number of patients included. In fact, pericardial effusion was detected in only 5 patients. Further study is necessary to verify the viability of this pocket-sized imaging device in diagnosing other cardiovascular diseases in clinical practice.

Finally, the Acuson P10 comprises the unit and the transducer. The size of the transducer is similar to those of current portable imaging devices, although the body of the system is pocket-sized. For future portable imaging devices, it would be beneficial if the transducer were miniaturized and combined into the body of the system.

\section{Conclusions}

This study demonstrated that the evaluation of cardiac chamber size and function by a pocket-sized portable echo- 
cardiography device was both feasible and accurate in clinical practice.

\section{Disclosure}

The authors have neither a conflict of interest nor financial disclosure.

\section{References}

1. Bruce CJ, Spittell PC, Montgomery SC, Bailey KR, Tajik AJ, Seward JB. Personal ultrasound imager: Abdominal aortic aneurysm screening. J Am Soc Echocardiogr 2000; 13: 674-679.

2. Vourvouri EC, Poldermans D, Schinkel AF, Koroleva LY, Sozzi FB, Parharidis GE, et al. Left ventricular hypertrophy screening using a hand-held ultrasound device. Eur Heart J 2002; 23: 1516-1521.

3. Spencer KT, Anderson AS, Bhargava A, Bales AC, Sorrentino M, Furlong K, et al. Physician-performed point-of-care echocardiography using a laptop platform compared with physical examination in the cardiovascular patient. J Am Coll Cardiol 2001; 37: 2013-2018.

4. Mangione S, Nieman LZ. Cardiac auscultatory skills of internal medicine and family practice trainees: A comparison of diagnostic proficiency. JAMA 1997; 278: 717-722.

5. Badgett RG, Lucey CR, Mulrow CD. Can the clinical examination diagnose left-sided heart failure in adults? JAMA 1997; 277: 1712 1719.

6. Lok CE, Morgan CD, Ranganathan N. The accuracy and interobserver agreement in detecting the 'gallop sounds' by cardiac auscultation. Chest 1998; 114: 1283-1288.

7. Egan M, Ionescu A. The pocket echocardiograph: A useful new tool? Eur J Echocardiogr 2008; 9: 721-725.

8. Lang RM, Bierig M, Devereux RB, Flachskampf FA, Foster E, Pellikka PA, et al. Recommendations for chamber quantification: A report from the American Society of Echocardiography's Guidelines and Standards Committee and the Chamber Quantification Writing Group, developed in conjunction with the European Association of Echocardiography, a branch of the European Society of Cardiology. $J$ Am Soc Echocardiogr 2005; 18: 1440-1463.

9. Bland JM, Altman DG. Statistical methods for assessing agreement between two methods of clinical measurement. Lancet 1986; 1: 307 310.

10. Wake R, Takeuchi M, Yoshiyama M, Yoshikawa J. Quantitative assessment of left ventricular function during contrast-enhanced dobutamine stress echocardiography predicts future cardiac events in diabetic patients. Circ J 2006; 70: 868-874.

11. Okura Y, Ohno Y, Ramadan MM, Suzuki K, Taneda K, Obata H, et al. Characterization of outpatients with isolated diastolic dysfunction and evaluation of the burden in a Japanese community: Sado Heart Failure Study. Circ J 2007; 71: 1013-1021.

12. Daimon M, Watanabe H, Abe Y, Hirata K, Hozumi T, Ishii K, et al. Normal values of echocardiographic parameters in relation to age in a healthy Japanese population: The JAMP study. Circ J 2008; 72: $1859-1866$.

13. Nishikage T, Nakai H, Lang RM, Takeuchi M. Subclinical left ventricular longitudinal systolic dysfunction in hypertension with no evidence of heart failure. Circ J 2008; 72: 189-194.

14. Vourvouri EC, Koroleva LY, Ten Cate FJ, Poldermans D, Schinkel $\mathrm{AF}$, van Domburg RT, et al. Clinical utility and cost effectiveness of a personal ultrasound imager for cardiac evaluation during consultation rounds in patients with suspected cardiac disease. Heart 2003; 89: $727-730$.

15. Greaves K, Jeetley P, Hickman M, Dwivedi G, Sabharwal N, Lim T, et al. The use of hand-carried ultrasound in the hospital setting: A cost-effective analysis. J Am Soc Echocardiogr 2005; 18: 620-625.

16. Black HR, Weltin G, Jaffe CC. The limited echocardiogram: A modification of standard echocardiography for use in the routine evaluation of patients with systemic hypertension. Am J Cardiol 1991; 67: $1027-1030$.

17. Kimura BJ, Pezeshki B, Frack SA, DeMaria AN. Feasibility of "limited" echo imaging: Characterization of incidental findings. J Am Soc Echocardiogr 1998; 11: 746-750.

18. Manasia AR, Nagaraj HM, Kodali RB, Croft LB, Oropello JM, KohliSeth R, et al. Feasibility and potential clinical utility of goal-directed transthoracic echocardiography performed by noncardiologist intensivists using a small hand-carried device (SonoHeart) in critically ill patients. J Cardiothorac Vasc Anesth 2005; 19: 155-159.

19. Schiller NB. Hand-held echocardiography: Revolution or hassle? J Am Coll Cardiol 2001; 37: 2023-2024.

20. Gorcsan J. Utility of hand-carried ultrasound for consultative cardiology. Echocardiography 2003; 20: 463-469.

21. Seward JB, Douglas PS, Erbel R, Kerber RE, Kronzon I, Rakowski H, et al. Hand-carried cardiac ultrasound (HCU) device: Recommendations regarding new technology: A report from the Echocardiography Task Force on New Technology of the Nomenclature and Standards Committee of the American Society of Echocardiography. J Am Soc Echocardiogr 2002; 15: 369-373. 